\title{
5 ' and 3' UTR thymidylate synthase polymorphisms modulate the risk of colorectal cancer independently of the intake of methyl group donors
}

\author{
BRUNO CARMONA ${ }^{1}$, CATARINA GUERREIRO ${ }^{1}$, MARÍLIA CRAVO ${ }^{2}$, \\ CARLOS NOBRE-LEITÃO ${ }^{2}$ and MIGUEL BRITO ${ }^{1}$
}

${ }^{1}$ Escola Superior de Tecnologia da Saúde de Lisboa, Av. D. João II, Lote 4.69.01, Parque das Nações, 1990-096 Lisboa;
${ }^{2}$ Instituto Português de Oncologia Francisco Gentil de Lisboa, Rua Prof. Lima Basto, 1093 Lisboa Codex, Portugal

Received May 5, 2008; Accepted June 9, 2008

DOI: $10.3892 / \mathrm{mmr} 00000023$

\begin{abstract}
Thymidylate synthase, as a rate-limiting step in DNA synthesis, catalyses the conversion of dUMP into dTMP using 5,10-methylenotetrahydrofolate as the methyl donor. Two polymorphisms have been described in this gene: a repeat polymorphism in the $5^{\prime}$ promoter enhancer region (3R versus $2 \mathrm{R}$ ) and a 6 bp deletion in the 3 ' unstranslated region. Both of these may affect protein levels. The present case control study was aimed at investigating the influence of these two polymorphisms on the development of colorectal cancer (CRC), as well as their potential interaction with folate, vitamin B6 and vitamin B12 intake. A total of 196 cases and 200 controls, matched for age and sex distribution, were included in the study. No association was found between CRC and the $28 \mathrm{bp}$ repeat polymorphism, but it was observed that individuals with the $6 \mathrm{bp} / \mathrm{del}$ and del/del genotypes had a significantly lower risk of developing the disease (OR=0.47; 95\% CI 0.30-0.72). A combined genotype (2R/2R; $6 \mathrm{bp} / \mathrm{del}+\mathrm{del} / \mathrm{del})$ was also found, which was associated with an even lower risk of developing of the disease $(\mathrm{OR}=0.42 ; 95 \%$ CI $0.26-0.69)$. No significant interaction between these polymorphisms and vitamin intake was observed. These results indicate for the first time that the $6 \mathrm{bp} / \mathrm{del}$ allele might be a protective factor in the development of CRC, independent of the intake of methyl group donors.
\end{abstract}

\section{Introduction}

Colorectal cancer (CRC) is today one of the most prevalent cancers in developed societies (1), representing 9.4\% of cancers in males and $10.1 \%$ in females (2). In Portugal, it is among

Correspondence to: Dr Miguel Brito, Escola Superior de Tecnologia da Saúde de Lisboa, Av. D. João II, Lote 4.69.01, Parque das Nações, 1990-096 Lisboa, Portugal

E-mail: miguel.brito@estesl.ipl.pt

Key words: thymidylate synthase polymorphisms, colorectal cancer, folate, vitamin B6, vitamin B12, methyl group donors the leading causes of cancer, and has one of the highest mortality rates in Europe $(3,4)$.

It is well known that the etiology of this tumour is multifactorial, involving both environmental and genetic factors $(5,6)$. One of the factors that has been associated with a decreased risk of CRC is the high consumption of folate (7-9). Several studies suggest an inverse association between folate status, either its intake or blood measurements, and the risk of this malignancy (10). The potential mechanisms whereby deficiency enhances and supplementation suppresses CRC have been extensively reviewed (11-13), and are related to the involvement of this vitamin in the mediation of the transfer of 1-carbon units (Fig. 1). Through this apparently simple function, folate plays a key role in a number of processes related to DNA integrity, such as DNA synthesis and DNA methylation (11-15). This explains why a number of in vitro and in vivo studies have shown that folate deficiency results in DNA strand breaks, DNA hypomethylation, impaired DNA repair and increased mutagenesis (11-15).

In a number of these studies, the observed inverse association between folate status and CRC risk was further modified by genetic polymorphisms of the enzymes involved in folate metabolism, namely methylene tetrahydrofolate reductase (MTHFR) and thymidylate synthase (TS). The former is an enzyme critical to folate metabolism that catalyses the irreversible conversion of 5,10-methyleneTHF into 5-methylTHF, thereby playing an important role in DNA methylation and synthesis (Fig. 1). A number of previous studies suggest that folate supplementation might be particularly effective in reducing the risk of $\mathrm{CRC}$ in subjects carrying the polymorphic allele C677T for MTHFR (16-19), as this polymorphism causes thermolability and reduced MTHFR activity. TS is the enzyme responsible for the conversion of deoxyuridine monophosphate (dUMP) into deoxythymidine monophosphate (dTMP), and competes with MTHFR for 5,10-methyleneTHF as the substrate of this methylation reaction (20). In respect to TS, a tandem repeat polymorphism has been identified in the 5'UTR enhancer region of the TS promoter that contains either triple (3R) or double $(2 \mathrm{R})$ repeats of a 28 -bp sequence (21). Individuals homozygous for triple repeats have TS mRNA levels 3.6 times higher than those homozygous for the double repeat genotype (22). Another 6-bp deletion in the 


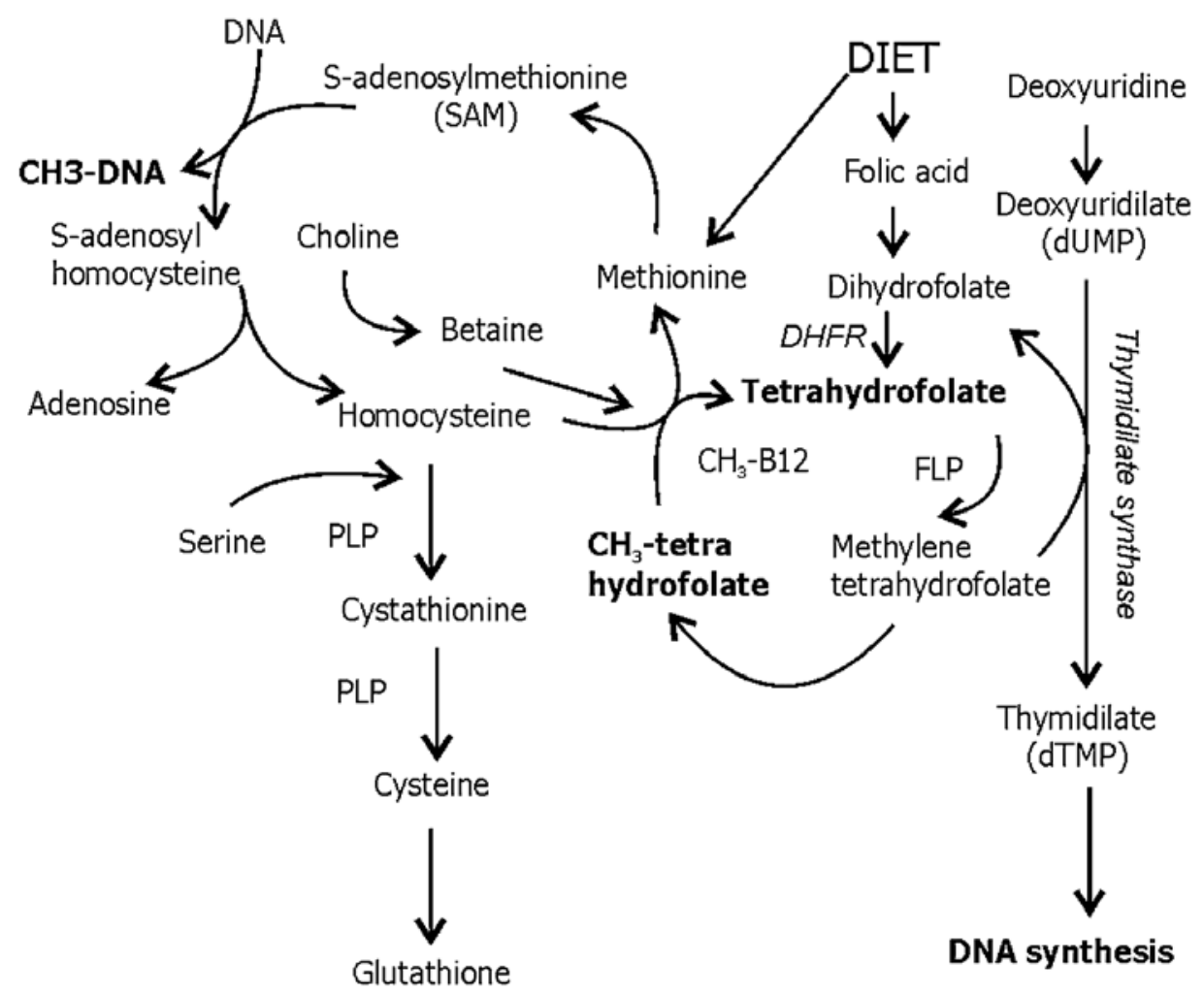

Figure 1. Folic acid cycle.

3'UTR of the TS gene has been identified, and previous studies have reported that tumour mRNA levels in patients with a homozygous wild-type genotype are 4.2-fold higher than those of patients with a homozygous variant genotype (23). Most studies have examined the importance of these polymorphisms in predicting either survival or response to 5-FU-based chemotherapy in tumours with a high expression of TS that are responding poorly to this agent and have a worse survival prognosis (24). To our knowledge, very few studies have examined whether these polymorphisms of the TS gene influence the risk of developing colorectal neoplasms. The results obtained from those that have been conducted are subject to some discrepancies (25-31).

The aim of this case-control study was to examine the potential influence of the two abovementioned TS UTR (untranslated region) polymorphisms on the development of CRC in a Portuguese population group, as well as to investigate potential interactions between these polymorphisms and the intake of methyl donor compounds, such as folate and vitamins B6 and B12.

\section{Materials and methods}

Study population. This case-control study was performed at the Instituto Português de Oncologia de Lisboa Francisco Gentil EPE (IPOLFG). The study was approved by the Scientific and Ethics committees of IPOLFG, and both patients and controls gave their informed written consent for participation.

The patient group comprised 196 subjects (104 males,

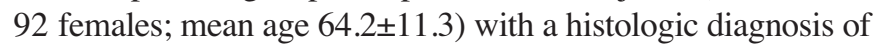
CRC, and the control group 200 healthy blood donor volunteers recruited from the same institute with a similar sex and age distribution (106 males, 94 females; mean age 62.2 \pm 12.1 ) and with no previous history of cancer at any site. Of the patients, 169/196 (86.5\%) had recently been diagnosed with CRC, while the remaining 27 (13.5\%) were being treated for disease relapse. In terms of previous therapies, 119/196 $(60.7 \%)$ had not received any form of treatment, 28/196 $(14.3 \%)$ had already been operated on, 13/196 (6.6\%) had undergone pelvic radiotherapy, 11/196 (5.6\%) had received one or more cycles of chemotherapy, and the remaining 25/196 $(12.8 \%)$ had undergone combined forms of treatment. TNM staging was as follows: stage I, 24/178 (13.5\%); stage II, 64/178 (35.9\%); stage III, 53/178 (29.8\%); stage IV, 37/178 $(20.8 \%)$. All subjects were Caucasian.

Nutritional evaluation. In order to quantify folate, vitamin B6 and vitamin B12 intake, we used a Food Frequency Questionnaire validated for a Portuguese population (32). Participants were asked to recall their habits in the year before CRC diagnosis (patients), or in the year prior to the interview (controls). Data on the type and quantity of food intake was then analysed using modified database Food Processor software, version 7 (Esha Research Inc. Salem, USA), which includes some Portuguese food items, allowing for the quantification of different macro- and micronutrients. Nutrient values were calculated from food and from supplements (taken by 11/196 of patients and by none of the controls).

Genotyping of polymorphisms. DNA was extracted from peripheral blood collected by digital puncture using the Generation Capture Card Kit (Gentra Systems). Fragments were amplified using specific primers for each allelic determination (5'-CCTGGCGCACGCTCTCTA-3' and 5'-GATCTG 
Table I. Mean intake of nutrients in the two population groups and odds ratio of the interaction between nutrient intake and colorectal cancer susceptibility.

\begin{tabular}{|c|c|c|c|c|c|}
\hline \multirow[b]{2}{*}{ Nutrients } & \multicolumn{2}{|c|}{ Mean intake $(\mu \mathrm{g} /$ day $)$} & \multicolumn{3}{|c|}{ Odds ratio $(95 \% \mathrm{CI})$} \\
\hline & Cases & Controls & Low & High & P-value \\
\hline Vitamin B6 & $2.83 \pm 1.06$ & $2.85 \pm 0.98$ & 1 & $0.71(0.52-1.07)$ & 0.10 \\
\hline Vitamin B12 & $14.53 \pm 9.05$ & $14.36 \pm 8.22$ & 1 & $0.89(0.59-1.34)$ & 0.58 \\
\hline Folic acid & $401.57 \pm 161.88$ & $433.40 \pm 161.32$ & 1 & $0.67(0.45-1.02)$ & 0.06 \\
\hline
\end{tabular}

Nutrient intake was divided in two groups: low, meaning intake lower than the calculated median, and high, meaning intake higher than the median. Control median intake of: vitamin B6, $2.82 \mu \mathrm{g}$ /day; vitamin B12, $12.63 \mu \mathrm{g} /$ day; folic acid, $422.22 \mu \mathrm{g} /$ day.

Table II. Allelic and genotypic distribution of the $28 \mathrm{bp}$ tandem repeat and $6 \mathrm{bp}$ deletion polymorphism alleles in the two population groups.

\begin{tabular}{|c|c|c|c|}
\hline & No. cases $(\%)$ & No. controls $(\%)$ & P-value \\
\hline \multicolumn{4}{|l|}{28 bp repeat } \\
\hline $2 \mathrm{R} / 2 \mathrm{R}$ & $24 \quad(13.9)$ & $30 \quad(17.6)$ & 0.330 \\
\hline $2 \mathrm{R} / 3 \mathrm{R}$ & $102 \quad(59.0)$ & $87 \quad(51.2)$ & \\
\hline $3 \mathrm{R} / 3 \mathrm{R}$ & $47 \quad(27.1)$ & $53 \quad(31.2)$ & \\
\hline $2 \mathrm{R}$ & $150(0.434)$ & $147(0.432)$ & 1.000 \\
\hline $3 R$ & $196(0.566)$ & $193(0.568)$ & \\
\hline \multicolumn{4}{|l|}{6 bp deletion } \\
\hline $6 \mathrm{bp} / 6 \mathrm{bp}$ & $99 \quad(53.5)$ & 72 (37.3) & 0.006 \\
\hline $6 \mathrm{bp} / \mathrm{del}$ & $69 \quad(37.3)$ & $100 \quad(51.8)$ & \\
\hline del/del & $17 \quad(9.2)$ & $21 \quad(10.9)$ & \\
\hline $6 \mathrm{bp}$ & $267(0.722)$ & $244(0.632)$ & $<0.010$ \\
\hline del & $103(0.278)$ & $142(0.368)$ & \\
\hline
\end{tabular}

Analysis performed using the $\chi^{2}$ test.

CCCCAGGTACTGC-3' for the 28 bp polymorphism; 5'-TTT CACAAGCTATTCCCTCAAA-3' and 5'-CAGAATGAA CAAAGCGTGGA-3' for the $6 \mathrm{bp}$ polymorphism). Both polymorphism fragments were amplified using $1.5 \mathrm{U}$ of Taq polymerase (Fermentas, Ontario, Canada) with a primer concentration of $200 \mathrm{nM}$ and a $\mathrm{Mg} 2^{+}$concentration of $2.5 \mathrm{mM}$. The 28 bp polymorphism fragment was amplified in the presence of $2 \mathrm{X}$ concentrated Enhancer Solution (Invitrogen, CA, USA). Amplification steps were as follows: $95^{\circ} \mathrm{C}$ for $10 \mathrm{~min}$, followed by 40 cycles at $95^{\circ} \mathrm{C}$ for $1 \mathrm{~min}, 60^{\circ} \mathrm{C}$ for $1 \mathrm{~min}$ and $72^{\circ} \mathrm{C}$ for $1 \mathrm{~min}$. A final extension step was performed at $72^{\circ} \mathrm{C}$ for $10 \mathrm{~min}$.

The 28 bp TS genotype was determined by separation of the fragments on $2 \%$ agarose gel. In the case of the 6 bp deletion genotype, Restriction Fragment Length Polymorphism (RFLP) analysis was used with $10 \mathrm{U}$ of DraI restriction enzyme (New England Biolabs, MA, USA) incubated at $37^{\circ} \mathrm{C}$ for $2 \mathrm{~h}$, followed by separation of the fragments on $4 \%$ agarose gel.
Statistical analysis. Odds ratio (OR) analysis was performed using binary logistic regression adjusted for sex, age and family history. Interaction between the polymorphisms and nutrient intake was evaluated by the likelihood ratio test. All analyses were performed using SPSS software, version 15.0.

\section{Results}

Of the total 196 patients and 200 controls included in this study, 173 patients and 170 controls were used in the analysis of the 28 bp repeat polymorphism, and 185 cases and 193 controls were used in the 6 bp deletion polymorphism. This was because certain DNA samples had failed to be amplified by PCR.

The mean daily intake of folate and vitamins B6 and B12 of the patients and controls is shown in Table I. To test whether the intake of these nutrients influenced the risk of developing CRC, patients and controls were respectively divided into two groups according to whether their intake was higher or lower than the calculated median of the control population, followed by an OR calculation (Table I). A daily intake of folate above $422.2 \pm 161.3 \mu \mathrm{g}$ /day was found to be associated with a $33 \%$ decreased risk of developing CRC $(\mathrm{OR}=0.67 ; 95 \% \mathrm{CI}$ 0.45-1.02). No significant differences were observed for vitamin B6 or B12.

Genotypic and allelic distributions of the 28 bp repeat polymorphism and the $6 \mathrm{bp}$ deletion polymorphism for both cases and controls are presented in Table II. In the $28 \mathrm{bp}$ repeat polymorphism, one sole individual was found to have four repeats. Since the functional consequences of this four repeat allele are not well known, the subject was excluded from the analysis. Regarding genotypic distribution, both cases and controls were in Hardy-Weinberg equilibrium for the two polymorphisms. No significant differences between cases and controls were observed in respect to the $28 \mathrm{bp}$ repeat polymorphism $(\mathrm{P}>0.05)$. In contrast, the $6 \mathrm{bp}$ deletion polymorphism was found to have a statistically significant $(\mathrm{P}<0.010)$ higher frequency of the del allele in controls as compared to cases. This finding strengthens the hypothesis that the presence of the del allele, in either heterozygosity or homozygosity, represents a protective effect. As shown in Table III, the $6 \mathrm{bp} / \mathrm{del}$ and del/del genotypes were observed to be associated with a reduction in the risk of $C R C(O R=0.47$; 95\% CI 0.30-0.72). However, the 28 bp repeat polymorphism showed no significant association with the risk of CRC $(\mathrm{OR}=1.33$; 95\% CI 0.74-2.39). 
Table III. Odds ratio analysis for the polymorphisms.

\begin{tabular}{lccc}
\hline & \multicolumn{3}{c}{ Odds ratio (95\% CI) } \\
\cline { 2 - 4 } Polymorphism & $2 \mathrm{R} / 2 \mathrm{R}$ & $2 \mathrm{R} / 3 \mathrm{R}+3 \mathrm{R} / 3 \mathrm{R}$ & P-value \\
\hline $28 \mathrm{bp}$ repeat & 1 & $1.29(0.71-2.36)$ & 0.41 \\
\hline & $6 \mathrm{bp} / 6 \mathrm{bp}$ & $6 \mathrm{bp} / \mathrm{del}+\mathrm{del} / \mathrm{del}$ & \\
\hline 6 bp deletion & 1 & $0.47(0.30-0.72)$ & 0.001 \\
\hline
\end{tabular}

Odds ratios were calculated using binary logistic regression adjusted for age, sex and family history.

OR analysis was performed in order to test whether there could be a combined genotype of this gene influencing CRC development. The analysis compared individuals homozygous for the $2 \mathrm{R}$ allele in the $28 \mathrm{bp}$ repeat polymorphism and those heterozygous or homozygous for the del allele in the $6 \mathrm{bp}$ deletion polymorphism $(2 \mathrm{R} / 2 \mathrm{R}, 6 \mathrm{bp} / \mathrm{del}+6 \mathrm{bp} / \mathrm{del})$ with the remaining individuals. It was observed that individuals carrying this combined genotype were at an even lower risk of developing $\mathrm{CRC}(\mathrm{OR}=0.42$; 95\% CI 0.29-0.73).

When the interaction between these polymorphisms and the intake of the three vitamins was tested, no significant associations were observed for either of the polymorphisms (data not shown). We also found no correlation between the polymorphisms and tumour location, disease staging, response to chemoradiotherapy or survival (data not shown).

\section{Discussion}

Folate, a water soluble B vitamin, has been shown to protect against cancer at several sites, including the colon $(11,13)$. Epidemiologic and clinical studies indicate that dietary folate intake and blood folate levels are inversely associated with the risk of developing both adenomas and CRC (7-9). Animal studies have also unequivocally established a causal relationship between folate deficiency and CRC (33). In a subsequent study (34), Kim et al demonstrated that the modulating effect of folate on CRC was dependent on both the timing and dose of folate intervention. More recently, some authors have raised the question of whether folate supplements taken by individuals already harbouring pre-malignant lesions can in fact increase the risk of CRC $(35,36)$. The mechanisms by which folate intake modulates the risk of malignancy are probably related to the fact that folate supplies the methyl group for either DNA methylation or for uracil conversion to thymidine $(10,12,37)$. Recent epidemiologic molecular studies have also suggested that the protective effect of folate could be further modified by interactions with genetic polymorphisms in the genes codifying the enzymes involved in folate metabolism. The most extensively studied genetic polymorphism is the C677T polymorphism of the MTHFR gene, which may influence the risk of CRC according to folate status, alcohol intake and the supply of methyl group donors, such as methionine, vitamins B6 and B12 $(13,38)$.
Most studies on TS, which converts dUMP to dTMP as a rate-limiting step in DNA synthesis, have examined the impact of the $28 \mathrm{bp}$ repeat and $6 \mathrm{bp} / \mathrm{del}$ polymorphisms on prognosis, or have evaluated them as indicators of response to 5-FU. Few studies have evaluated the influence of these polymorphisms on the aetiology of CRC, and those that have done have achieved discrepant results. For example, in respect to colorectal adenomas, which are the precursor lesions for CRC, Ulrich et al (23) observed that the TS $28 \mathrm{bp}$ tandem repeat polymorphism was not an independent risk factor for colorectal adenomas. However, there was a statistically significant genenutrient interaction with dietary folate intake: in individuals with the $3 \mathrm{R} / 3 \mathrm{R}$ genotype, folate intake over $440 \mu \mathrm{g} /$ day was associated with decreased risk, whereas in individuals with the $2 \mathrm{R} / 2 \mathrm{R}$ variant, a 1.5 -fold increased risk was observed with high folate intake. In the same study, the TS 6 bp deletion polymorphism was not demonstrated to modify the risk for colorectal adenomas, nor was any association with folate intake found. In contrast, in a case-control study nested within a prospective Health Professionals Follow-up Study, Chen et al (39) observed significant interaction between the TS 28 bp promoter polymorphism and alcohol intake, which modulated the risk of developing colorectal adenomas. The effect of the $6 \mathrm{bp} / \mathrm{del}$ polymorphism was not examined in that study (39).

As regards the influence of these polymorphisms on CRC development Ulrich et al observed that men carrying the 2R/2R genotype had a $30 \%$ decreased risk of CRC compared to those with the homozygous $3 \mathrm{R}$ genotype. No association was found for the 6 bp polymorphism (31). Chen et al (40), using the same study design within the prospective Physicians' Health Study, observed no association between the risk of $\mathrm{CRC}$ and the $6 \mathrm{bp} / \mathrm{del}$ polymorphism, However, in regard to the 28 bp polymorphism, individuals with the $2 \mathrm{R}$ allele had a non-significant decreased risk of CRC compared to those with the $3 R / 3 R$ genotype. This suggests a protective effect for the $2 \mathrm{R}$ allele. Additionally, none of the compound genotypes significantly influenced the risk of CRC, nor was any modulating effect of folate status observed. These results are in only partial agreement with the current findings. This study also demonstrated that folate intake per se had a protective effect against the risk of developing CRC (OR=0.67; 95\% CI 0.45-0.99). Our findings with respect to TS polymorphisms were similar to those of Chen et al (40) and Ulrich et al (31), in that differences regarding the 28 bp polymorphism were non-significant. However, in contrast with the results obtained by Ulrich et al (31), who found that the TS 6 bp polymorphic allele was not associated with a decreased risk of CRC, in the present study individuals homozygous or heterozygous for this variant carried a significantly and unequivocally lower risk of developing CRC. Furthermore, by combining the protective effects of the $6 \mathrm{bp} / \mathrm{del}$ allele and the $28 \mathrm{bp} 2 \mathrm{R}$ allele, we found a combined genotype of the TS gene (2R/2R, 6 bpdel/del+ 6 bpdel), which was associated with a $58 \%$ decreased risk of developing CRC. In agreement with Chen et al (40), no significant interactions between these genetic polymorphisms and folate intake were observed in the present study.

The reasons for the discrepancies observed among these studies are not readily apparent. However, it is worth noting that all previous studies on the influence of these genetic poly- 
morphisms on the risk of developing colorectal adenomas or CRC were performed in North America, where the diet is certainly very different from the average dietary intake in Portugal. One could also speculate that interactions with other genetic polymorphisms could explain these differing results. However, no statistically significant differences were found between the genotypic distribution of the present study and that of others $(31,40)$, with the exception of the genotypic distribution of the $28 \mathrm{bp}$ repeat allele compared to the cancer population used by Ulrich et al $(\mathrm{P}<0.05)$ (data not shown) (31). In a previous study using the same population as the current study, we analysed polymorphisms in folate metabolizing enzymes involved in the DNA methylation cycle, including C677T polymorphisms of methylene terahydrofolate reductase (MTHFR). No interaction was observed between the two polymorphisms of TS analysed in the present study and the C677T polymorphism of MTHFR in terms of modulating the risk of CRC (unpublished results).

Altogether, the findings of this and previous studies $(31,40)$ suggest that alleles resulting in decreased gene expression exhibit a protective effect against the development of CRC. As these polymorphisms contribute to the final intracellular concentration of TS protein, we further investigated the role of two combined genotypes $(2 \mathrm{R} / 2 \mathrm{R}+6 \mathrm{bp} / \mathrm{del}$, del/del) in the development of CRC. Since the $2 \mathrm{R}$ allele is associated with a decreased translation level of the TS mRNA, and the del allele with TS mRNA instability, individuals with these combined genotypes should have a decreased TS protein level. Indeed, such individuals were found to have a $2.4 \mathrm{x}$ reduction in the risk of developing CRC. It is therefore safe to hypothesize that low TS protein levels lead to a reduction in the risk of developing CRC. This may be due to the fact that TS and MTHFR compete for the same substrate $(5,10$ methyleneTHF). High TS levels would lead to a depletion of the 5,10-methyleneTHF pool, which could impair the conversion of homocysteine into methionine (Fig. 1). Methionine is the immediate precursor of S-Adenosylmethionine, the universal methyl donor for several compounds, including DNA. Thus, at least theoretically, elevated TS activity could impair DNA methylation, which has been widely recognized as an important epigenetic mechanism for the control of gene transcription. This hypothesis needs to be further confirmed, and is currently being tested.

To the best of our knowledge, this is the first study performed in a southern European country demonstrating a protective effect for both the $6 \mathrm{bp} / \mathrm{del}$ allele per se, as well as for a compound genotype including this allele and the $2 \mathrm{R}$ allele from the $28 \mathrm{bp}$ polymorphism. These protective effects do not appear to be mediated by folate or by other methyl group donor intake.

Although future studies are required to elucidate why the same polymorphisms have differing roles in different populations, our results contribute to the understanding of the complex interaction between genes and nutrients in the modulation of cancer risk.

\section{Acknowledgements}

This work was funded by a grant from the Fundação Calouste Gulbenkian No. 68925/2005.

\section{References}

1. Wilmink ABM: Overview of the epidemiology of colorectal cancer. Dis Colon Rectum 40: 483-493, 1997.

2. Boyle P and Langman JS: ABC of colorectal cancer: epidemiology. BMJ 321: 805-808, 2000.

3. Bingham S and Riboli E: Diet and cancer - the European Prospective Investigation into Cancer and Nutrition. Nat Rev Cancer 4: 206-215, 2004.

4. Pinheiro PS, Tyczynski JE, Bray F, Amado J, Matos E and Parkin DM: Cancer incidence and mortality in Portugal. Eur J Cancer 39: 2507-2520, 2003.

5. Campos FG, Logullo Waitzberg AG, Kiss DR, Waitzberg DL, Habr-Gama A and Gama-Rodrigues J: Diet and colorectal cancer: current evidence for aetiology and prevention. Nutr Hosp 20: $18-25,2005$.

6. Willett W: The search for the causes of breast and colon cancer. Nature 338: 389-394, 1989

7. Sanjoaquin MA, Appleby PN, Thorogood M, Mann J and Key TJ: Nutrition, lifestyle and colorectal cancer incidence: a prospective investigation of 10998 vegetarians and non-vegetarians in the United Kingdom. Br J Cancer 90: 118-121, 2004.

8. Terry P, Giovannucci E, Michels KB, Bergkvist L, Hansen H, Holmberg L and Wolk A: Fruit, vegetables, dietary fiber, and risk of colorectal cancer. J Natl Cancer Inst 93: 525-533, 2001.

9. Thun MJ, Calle EE, Namboodiri MM, et al: Risk factors for fatal colon cancer in a large prospective study. J Natl Cancer Inst 84: 1491-1500, 1992

10. Zhang SM, Moore SC, Lin J, Cook NR, Manson JE, Lee IM and Buring JE: Folate, vitamin B6, multivitamin supplements, and colorectal cancer risk in women. Am J Epidemiol 163: 108-115, 2006.

11. Choi S-W and Mason JB: Folate status: effects on pathways of colorectal carcinogenesis. J Nutr 132: S2413-S2418, 2002.

12. Fenech M: The role of folic acid and vitamin B12 in genomic stability of human cells. Mutat Res 475: 57-67, 2001.

13. Kim YI: Folate and carcinogenesis: evidence, mechanisms, and implications. J Nutr Biochem 10: 66-88, 1999.

14. Ames BN: DNA damage from micronutrient deficiencies is likely to be a major cause of cancer. Mutat Res 475: 7-20, 2001.

15. Duthie SJ: Folic acid deficiency and cancer: mechanisms of DNA instability. Br Med Bull 55: 578-592, 1999.

16. Chen J, Giovannucci E, Kelsey K, et al: A methylenetetrahydrofolate reductase polymorphism and the risk of colorectal cancer. Cancer Res 56: 4862-4864, 1996.

17. Ma J, Stampfer MJ, Giovannucci E, et al: Methylenetetrahydrofolate reductase polymorphism, dietary interactions, and risk of colorectal cancer. Cancer Res 57: 1098-1102, 1997.

18. Slattery ML, Potter JD, Samowitz W, Schaffer D and Leppert M: Methylenetetrahydrofolate reductase, diet, and risk of colon cancer. Cancer Epidemiol Biomarkers Prev 8: 513-518, 1999.

19. Ulrich CM, Kampman E, Bigler J, et al: Colorectal adenomas and the C677T MTHFR polymorphism: evidence for gene-environment interaction? Cancer Epidemiol Biomarkers Prev 8: 659-668, 1999.

20. Sharp L and Little J: Polymorphisms in genes involved in folate metabolism and colorectal neoplasia: a HuGE review. Am J Epidemiol 159: 423-443, 2004

21. Takeishi K, Kaneda S, Ayusawa D, Shimizu K, Gotoh O and Seno T: Nucleotide sequence of a functional cDNA for human thymidylate synthase. Nucl Acids Res 13: 2035-2043, 1985.

22. Pullarkat ST, Stoehlmacher J, Ghaderi V, et al: Thymidylate synthase gene polymorphism determines response and toxicity of 5-FU chemotherapy. Pharmacogenomics J 1: 65-70, 2001.

23. Ulrich CM, Bigler J, Bostick R, Fosdick L and Potter JD: Thymidylate synthase promoter polymorphism, interaction with folate intake, and risk of colorectal adenomas. Cancer Res 62: 3361-3364, 2002.

24. Salonga D, Danenberg KD, Johnson M, et al: Colorectal tumors responding to 5-fluorouracil have low gene expression levels of dihydropyrimidine dehydrogenase, thymidylate synthase, and thymidine phosphorylase. Clin Cancer Res 6: 1322-1327, 2000.

25. Curtin K, Ulrich CM, Samowitz W, Bigler J, Caan B, Potter JD and Slattery ML: Thymidylate synthase polymorphisms and colon cancer: associations with tumor stage, tumor characteristics and survival. Int J Cancer 120: 2226-2232, 2007.

26. Dotor E, Cuatrecases M, Martinez-Iniesta M, et al: Tumor thymidylate synthase 1494del6 genotype as a prognostic factor in colorectal cancer patients receiving fluorouracil-based adjuvant treatment. J Clin Oncol 24: 1603-1611, 2006. 
27. Hitre E, Budai B, Adleff V, et al: Influence of thymidylate synthase gene polymorphisms on the survival of colorectal cancer patients receiving adjuvant 5-fluorouracil. Pharmacogenet Genomics 15: 723-730, 2005.

28. Marsh S and McLeod HL: Thymidylate synthase pharmacogenetics in colorectal cancer. Clin Colorectal Cancer 1: 175-178, 2001.

29. Marsh S, McKay JA, Cassidy J and McLeod HL: Polymorphism in the thymidylate synthase promoter enhancer region in colorectal cancer. Int J Oncol 19: 383-386, 2001.

30. Tsuji T, Hidaka S, Sawai T, et al: Polymorphism in the thymidylate synthase promoter enhancer region is not an efficacious marker for tumor sensitivity to 5-fluorouracil-based oral adjuvant chemotherapy in colorectal cancer. Clin Cancer Res 9: 3700-3704, 2003.

31. Ulrich CM, Curtin K, Potter JD, Bigler J, Caan B and Slattery ML: Polymorphisms in the reduced folate carrier, thymidylate synthase, or methionine synthase and risk of colon cancer. Cancer Epidemiol Biomarkers Prev 14: 2509-2516, 2005.

32. Lopes C: Nutrition and acute myocardial infarction: community case control study. PhD Thesis, College of Medicine, Porto University, Portugal, 2000.

33. Cravo ML, Mason JB, Dayal Y, Hutchinson M, Smith D, Selhub J and Rosenberg IH: Folate deficiency enhances the development of colonic neoplasia in dimethylhydrazine-treated Rats. Cancer Res 52: 5002-5006, 1992.
34. Kim YI, Salomon RN, Graeme-Cook F, Choi SW, Smith DE, Dallal GE and Mason JB: Dietary folate protects against the development of macroscopic colonic neoplasia in a dose responsive manner in rats. Gut 39: 732-740, 1996.

35. Mason JB, Dickstein A, Jacques PF, Haggarty P, Selhub J, Dallal G and Rosenberg IH: A temporal association between folic acid fortification and an increase in colorectal cancer rates may be illuminating important biological principles: a hypothesis. Cancer Epidemiol Biomarkers Prev 16: 1325-1329, 2007.

36. Ulrich CM and Potter JD: Folate and cancer - timing is everything. JAMA 297: 2408-2409, 2007.

37. Otani T, Iwasaki M, Hanaoka T, et al: Folate, vitamin B6, vitamin $\mathrm{B} 12$, and vitamin $\mathrm{B} 2$ intake, genetic polymorphisms of related enzymes, and risk of colorectal cancer in a hospitalbased case-control study in Japan. Nutr Cancer 53: 42-50, 2005.

38. Kim YI: Methylenetetrahydrofolate reductase polymorphisms, folate, and cancer risk: a paradigm of gene-nutrient interactions in carcinogenesis. Nutr Rev 58: 205-209, 2000.

39. Chen J, Kyte C, Chan W, Wetmur JG, Fuchs CS and Giovannucci E: Polymorphism in the thymidylate synthase promoter enhancer region and risk of colorectal adenomas. Cancer Epidemiol Biomarkers Prev 13: 2247-2250, 2004.

40. Chen J, Hunter DJ, Stampfer MJ, et al: Polymorphism in the thymidylate synthase promoter enhancer region modifies the risk and survival of colorectal cancer. Cancer Epidemiol Biomarkers Prev 12: 958-962, 2003. 